\title{
Quantitative EEG reflects Non-Dopaminergic Disease Severity in Parkinson's Disease
}

Geraedts, V.J. MD MSc ${ }^{\text {a, b }}$, Marinus, J. PhD ${ }^{\text {a }}$, Gouw A.A.MD PhD ${ }^{\text {b }}$, Mosch, A. MD PhD ${ }^{\text {c }}$, Stam, C.J. MD PhD ${ }^{b}$, van Hilten J.J. MD PhD ${ }^{\text {a }}$, Contarino, M.F. MD PhD ${ }^{a, c^{*}}$, Tannemaat, M.R. MD PhD ${ }^{\text {a }}$

Affiliations:

a. Department of Neurology, Leiden University Medical Centre, Albinusdreef 2, 2333ZA Leiden, the Netherlands.

b. Department of Clinical Neurophysiology and MEG Center, Neuroscience Campus Amsterdam, VU University Medical Center, PO Box 7057, 1007 MB Amsterdam, the Netherlands.

c. Department of Neurology, Haga Teaching Hospital, Els Borst-Eilersplein 275, 2545 AA Den Haag, the Netherlands.

\section{Running-title: EEG reflects non-dopaminergic disease severity}

Abstract word count: 200

Manuscript word count: 3164

Number of tables: 1

Number of figures: 3

Number of supplementary tables: 3

Number of supplementary figures: 2

Conflicts of interest concerning the research: none.

Funding sources for study: this work was supported by a grant from the 'Stichting ParkinsonFonds' and the 'Stichting Alkemade-Keuls'.

Corresponding author:

*M.F. Contarino, Department of Neurology, Leiden University Medical Center, Albinusdreef 2, 2333 ZA Leiden, The Netherlands (tel.: +31 71526 3964; fax: +31 71524 8253; e-mail: m.f.contarino@lumc.nl) 


\section{Highlights}

- EEG parameters correlate to measures of disease severity in patients with Parkinson's Disease.

- Both EEG slowing and reduced connectivity correlate to non-dopaminergic disease severity.

- The proposed markers may be useful in the screening process for Deep Brain Stimulation.

\section{Abstract}

OBJECTIVE In Parkinson's Disease (PD), measures of non-dopaminergic systems involvement may reflect disease severity and therefore contribute to patient-selection for Deep Brain Stimulation (DBS). There is currently no determinant for non-dopaminergic disease severity. In this exploratory study, we investigated whether quantitative EEG reflects non-dopaminergic disease severity in PD.

METHODS Sixty-three consecutive PD patients screened for DBS were included (mean age $62.4 \pm$ 7.2 years, $32 \%$ females). Relative spectral powers and the Phase-Lag-Index (PLI) reflecting functional connectivity were analysed on routine EEGs. Non-dopaminergic disease severity was quantified using the SENS-PD score and its subdomains; motor-severity was quantified using the MDS-UPDRS III.

RESULTS The SENS-PD composite score correlated with a spectral ratio $((\delta+\theta) /(\alpha 1+\alpha 2+\beta)$ powers) (global spectral ratio Pearson's $r=0.4,95 \%$ Confidence Interval $(95 \% \mathrm{CI}) 0.1$ to 0.6 ), and PLI in the $\alpha 2$ band $(10-13 \mathrm{~Hz})(\mathrm{r}=-0.3,95 \% \mathrm{CI}-0.5$ to -0.1$)$. These correlations seem driven by the subdomains cognition and psychotic symptoms. MDS-UPDRS III was not significantly correlated with EEG parameters.

CONCLUSIONS EEG slowing and reduced functional connectivity in the $\alpha 2$ band were associated with non-dopaminergic disease severity in PD.

SIGNIFICANCE The described EEG parameters may have complementary utility as determinants of non-dopaminergic involvement in PD.

KEYWORDS: 'quantitative EEG', 'Parkinson's Disease', 'Deep Brain Stimulation', 'connectivity', 'non-motor severity' 


\section{Introduction}

Parkinson's Disease (PD) is a multisystem neurodegenerative disorder, caused by progressive degeneration of both dopaminergic and non-dopaminergic neurons (Jellinger, 2012). Dopaminergic neurons account primarily for the characteristic motor symptoms of PD, whilst non-dopaminergic neurons account for non-motor symptoms such as impaired cognition, psychiatric manifestations or sleep disturbances. PD is typically treated with oral dopaminergic medication, which alleviates motor symptoms. However, medication-related motor complications occur in the majority of patients within 10 years of disease (Ahlskog et al. , 2001). Patients refractory to oral treatment may be eligible for Deep Brain Stimulation (DBS), which ameliorates motor complications and improves quality of life (Deuschl et al. , 2013). DBS is particularly effective in patients perceiving substantial motor improvement of upon dopaminergic treatment (Moldovan et al. , 2015). However, non-dopaminergic symptoms such as cognitive impairment,(Contarino et al. , 2007) depression (Weaver et al. , 2009), speech intelligibility (Tripoliti et al. , 2011) and axial symptoms (Russmann et al. , 2004) may deteriorate post-DBS. This indicates the need for accurate assessment of non-dopaminergic disease severity during the preoperative selection process.

Clinical, neuropsychological and psychiatric evaluations are used to rule out severe cognitive decline or psychiatric comorbidity. However, several factors including intelligence, education, and personality limit the interpretability of clinimetric assessments (Duncan, 1993). Moreover, questionnaires and performance tasks are susceptible to misinterpretation, social desirability bias, or fatigue (Duckworth et al. , 2015). Therefore, there is a need for complementary measures reflecting disease severity in PD to aid the identification of DBS candidates.

Quantitative Electroencephalography (qEEG) is an inexpensive and widely available tool which measures brain activity directly. Previous studies applied qEEG to examine clinical domains in PD, such as cognition (Caviness et al. , 2015, Cozac et al. , 2016), response to treatment (George et al. , 2013) or motor impairment (Babiloni et al. , 2011, George et al. , 2013). Global oscillatory slowing of the EEG spectrogram is a highly suitable biomarker for cognitive impairment in PD (Caviness et al. , 2015). Recent advances in neurophysiology have provided more complex markers such as 
connectivity parameters and graph theory estimations, which quantify brain network disorganization.

The Phase-Lag Index (PLI), which reflects functional connectivity, was suggested as a potential biomarker of PD dementia (Utianski et al. , 2016). To our knowledge the relation of qEEG parameters to measures of non-dopaminergic severity in PD has not been investigated so far. We aimed to investigate whether qEEG correlates with clinical measures of disease severity, in order to ultimately provide neurophysiological determinants of disease severity.

\section{Methods}

\subsection{Study participants}

All consecutive PD patients who were referred for preoperative screening to the DBS centre of Leiden University Medical Center (LUMC) and Haga Teaching Hospital between September 2015 and July 2017 were included in the study. All patients fulfilled the Movement Disorders Society PD criteria for clinically established PD (Postuma et al. , 2015). Written informed consent was obtained from all patients. A formal ethical evaluation of this study was waived by the local medical ethics committee.

\subsection{Outcome measures}

Motor function was assessed with the Movement Disorders Society Unified Parkinson’s Disease Rating Scale (MDS-UPDRS) part III (Goetz et al. , 2007) (range 0-132), whereas the SEverity of Non-dopaminergic Symptoms in Parkinson’s Disease (SENS-PD) scale quantified non-dopaminergic disease severity (van der Heeden et al. , 2016) (range 0-54). The SENS-PD scale is a composite score comprising three items with four response options (range 0-3) from each of the following six predominantly non-dopaminergic domains: postural instability and gait difficulty (PIGD), psychotic symptoms, excessive daytime sleepiness (EDS), autonomic dysfunction, cognitive impairment and depressive symptoms (van der Heeden et al. , 2016). These six domains represent a coherent complex of symptoms that is already present in early disease stages and increases in severity with age and advancing disease. The SENS-PD scale is a recently developed, short, reliable and valid scale that includes symptoms that do not improve with dopaminergic medication and may therefore more 
accurately reflect severity and progression of the underlying disease than currently used dopaminesensitive measures. Higher scores on both the MDS-UPDRS III and SENS-PD scale reflect more severe impairment.

Patients were subdivided a posteriori into three groups of comparable size, according to the tertile in which their SENS-PD score fell: low disease severity (range 4-10), intermediate severity (range 1114) and high severity (range 15-24).

\subsection{EEG recording and pre-processing}

EEGs were recorded with patients lying supine, with eyes closed, during a state of relaxed wakefulness. Light was kept at moderate intensity. $\mathrm{Ag} / \mathrm{AgCl} \mathrm{EEG}$ electrodes were placed on the scalp using 21 standard 10-20 EEG electrode positions. Additional ECG and horizontal eye movement leads were added for identification of artifacts. Data were acquired online using a Nihon Kohden EEG-1200 system, with a $500 \mathrm{~Hz}$ sampling rate, a 16-bit analog-to-digital converter, and band-filtered between 0.16 and $70 \mathrm{~Hz}$. An EEG technician monitored signal quality throughout the entire recording; patients were alerted by acoustic stimuli upon drowsiness. All patients used dopaminergic medication according to their individual schedule.

\subsection{EEG analysis}

EEG data were re-referenced towards a source derivation which approaches the surface Laplacian derivation (Hjorth, 1980). Five consecutive, non-overlapping 4096-point epochs lasting 8.192 seconds were selected for further analysis after an artifact-free signal was visually confirmed. The individual epochs were offline converted to American Standard Code for Information Interchange (ASCII) format and further analysed using Brainwave software (BrainWave version 0.9.152.4.1, C.J. Stam; available at http://home.kpn.nl/stam7883/brainwave.html). Recordings with less than five artifact-free epochs were excluded from analysis. Analyses were performed in signal space to allow for direct analysis of the raw EEG data in Brainwave software, which is easily reproducible for most clinicians and may increase the utility of our findings. 
Spectral analysis was performed off-line by processing each epoch with a Fast Fourier Transform (FFT) and averaged to produce a power spectrum for each individual electrode. The frequency bands were defined as $\delta(0.5-4.0 \mathrm{~Hz}), \theta(4.0-8.0 \mathrm{~Hz}), \alpha 1(8.0-10.0 \mathrm{~Hz}), \alpha 2(10.0-13.0 \mathrm{~Hz})$ and $\beta(13.0$ $-30.0 \mathrm{~Hz}) . \gamma$ band power was not analysed due to frequent contamination with muscle artifacts. Relative bandpower was calculated by dividing the absolute bandpower of each frequency band by the total absolute bandpower from the FFT average per channel. Regional band powers were defined as: frontal for F3, F4, F7, F8 and Fz, temporal for T3, T4, T5 and T6, parietal for P3, P4 and Pz, central for $\mathrm{C} 3, \mathrm{C} 4$ and $\mathrm{Cz}$, and occipital for $\mathrm{O} 1$ and $\mathrm{O} 2$. Global bandpower was defined as the average of all regional band powers. A spectral ratio was calculated by dividing the sum of the relative power in the $\delta$ and $\theta$ bands by the sum of the relative power in the $\alpha$ and $\beta$ bands. These spectral bands were selected a priori to incorporate as much of the EEG spectrum as possible, without contaminating the results with possible artefacts that may be present in the fast frequency bands.

Functional connectivity was assessed by calculating the PLI in each frequency band. The PLI quantifies phase coupling (range 0-1) whilst being insensitive to common sources and volume conduction. $\mathrm{PLI}=0$ indicates either no phase synchronization or equal in both leading and lagging throughout the epoch, PLI=1 indicates perfect phase-locking (Stam et al. , 2007).

\subsection{Statistical analysis}

Baseline demographic, clinical, and test variables were not-normally distributed and were thus compared between the three groups with Kruskal-Wallis $\mathrm{H}$ tests for continuous variables and Pearson's $\chi^{2}$ tests for dichotomous variables. Included and excluded patients were compared using Mann-Whitney U tests. QEEG parameters were compared across SENS-PD tertiles using KruskalWallis $\mathrm{H}$ tests; Mann-Whitney U tests compared the high- and low-disease severity tertiles. The association between the SENS-PD composite score, SENS-PD subdomains, MDS-UPDRS III score and qEEG parameters was assessed by calculating partial Pearson's $r$ correlation coefficients, with an additional correction for age and usage of psychoactive medication, which may influence EEG parameters. Bias-corrected and accelerated bootstrapping was performed with 7000 samples to normalize the data; further increasing the amount of samples did not improve statistical accuracy. 
162 Given the exploratory nature of our study, to avoid type II errors, no further correction for multiple

163

164

165

166

167 testing was applied. Missing values were imputed using multiple imputation with five iterations, if no more than $15 \%$ of the data was missing.

All analyses were performed using IBM Statistical Package for the Social Sciences 23 Software (SPSS Inc., Chicago, Illinois, USA). Significance was set at the 0.05 level. Graphical visualization of results was performed in either MATLAB R2016A (The MathWorks Inc., Natick, Massachusetts, USA) or GraphPad Prism 7.02 (GraphPad Software Inc., La Jolla, California, USA).

\section{Results}

\subsection{Patient characteristics}

Eighty patients underwent DBS screening during the study period. Seventeen patients were excluded due to gross artifacts during EEG recordings (low disease severity: $n=8$, intermediate severity: $n=3$, high disease severity: $n=6$ ); analysis was thus performed on 63 patients ( $32 \%$ female). There were no significant differences in demographic and clinical variables between included and excluded patients. Mean (SD) age was 62.4 (7.2) years, and disease duration 11.9 (6.3) years (table 1). There were no significant differences in age, sex, disease duration, psychoactive drug usage and MDS-UPDRS III score between the three SENS-PD tertiles. By design, both the SENS-PD score and all six subdomains differed significantly between tertiles, with higher scores for the high-disease-severity group.

\subsection{Spectral analyses}

Slower EEG frequency bandpowers (i.e. $\delta$ and $\theta$ ) were higher in the high-disease-severity group, whereas faster frequency bandpowers (i.e. $\alpha 1, \alpha 2$ and $\beta$ ) were higher in the low-disease-severity group (Figure 1).

Mean spectral ratios (supplementary table 1) differed significantly between the high and low tertiles, both globally and over the frontal, central, parietal and occipital regions. The high-disease-severity group demonstrated a higher spectral ratio, indicating a greater proportion of slow EEG power. 
The SENS-PD composite score showed a significant positive correlation with the spectral ratio: a higher ratio, reflecting a greater proportion of slow EEG power, correlated with more severe nondopaminergic impairment, across all brain regions. The subdomains cognition and psychotic symptoms showed significant correlations for all regions, whilst the subdomains PIGD and depression showed a non-significant positive trend. The subdomains EDS, autonomic dysfunction and the MDSUPDRS III score did not correlate with EEG parameters (Figure 2).

\subsection{Functional connectivity}

Global PLI in the $\alpha 2$ band was significantly different between the three tertiles, while differences in the other frequency bands were non-significant (supplementary table 2). Regional mean $\alpha 2$ PLI values (supplementary table 3) differed significantly between the tertile groups over the frontal, central, temporal and occipital regions. Patients in the high-disease-severity group had a lower connectivity density, i.e. number of connections per threshold PLI value, than patients in the low-disease-severity group (Figure 3), indicating that higher disease severity was associated with lower functional connectivity.

Global, frontal, central, parietal and occipital PLI in the $\alpha 2$ band was significantly negatively associated with the SENS-PD composite score and with the cognitive subdomain (supplementary figures 1 and 2). The subdomain 'psychotic symptoms' showed a significant negative correlation over the central electrodes and a negative trend over all brain regions. In all instances, reduced functional connectivity correlated with higher disease severity.

\section{Discussion}

Several qEEG parameters were found to have potential as neurophysiological determinants of advanced non-dopaminergic disease severity in PD. As high non-dopaminergic disease severity is a relative contra-indication for DBS, qEEG analysis may ultimately complement clinimetric evaluations to optimize the screening process of DBS candidates. 
210 Slower EEG oscillatory activity was associated with more advanced non-dopaminergic disease

211 severity measured by the SENS-PD score and, in particular, with the subdomains cognition and

212 psychotic symptoms, with a trend towards a correlation with PIGD and depressive symptoms.

213 Conversely, the subdomains EDS and autonomic dysfunction did not show a clear pattern of

214 correlation. Motor impairment did not significantly correlate with spectral parameters.

Previous literature confirms the association between cortical slowing and cognitive impairment in PD (Caviness et al. , 2015). The consistent EEG slowing both on a global level and across all the different brain areas indicates diffuse dysfunction. It has been previously suggested that diffuse cortical slowing in PD reflects a degeneration of a non-dopaminergic system with ascending cortical projections (Olde Dubbelink et al. , 2013), which seems compatible with the observed correlation between clinical measures of non-dopaminergic disease severity and EEG slowing. Cortical slowing has been associated with severity of psychiatric symptoms also in other conditions. In schizophrenic patients, auditory hallucinations were associated with task-related $\theta$ slowing (Zheng et al. , 2015) and in cases with a delirium, visual hallucinations were associated with $\theta-\delta$ slowing (Teeple et al. , 2009). Although results of these studies based on other diseases may not be directly comparable with $\mathrm{PD}$, we speculate that these findings suggest a more general cortical desynchronization, rather than network alterations that are specific to PD. Reduced functional connectivity in the $\alpha 2$ band significantly correlated with higher non-dopaminergic disease severity, particularly with cognition, with a similar trend for the subdomains psychotic symptoms, depression, PIGD and EDS. Motor impairment did not correlate with functional connectivity. Only one EEG-study previously investigated PLI in PD, reporting reduced $\alpha 1$ PLI values in demented PD patients relative to cognitively normal PD patients. $\alpha 1$ PLI likewise correlated with the MMSE scale (Utianski et al. , 2016). Reduced $\alpha 1$ PLI values were also found to correlate with increased severity in Alzheimer's Disease (AD) (Engels et al. , 2015). Our results indicate a nonsignificant trend for $\alpha 1$ PLI, whereas we found $\alpha 2$ PLI to be significantly reduced. This discrepancy can be explained by a difference in population, as previous studies investigated patients with more advanced cognitive deterioration while our population did not include demented PD patients, being this a contra-indication for the DBS screening procedure. However, these findings suggest that 
desynchronization of a similar $\alpha$-band (either $\alpha 1$ or $\alpha 2$ ) network might underlie cognitive deterioration in both PD and AD.

The described qEEG parameters, both oscillatory slowing and functional connectivity, demonstrate discriminative ability at a group-level and may be further investigated to determine their potential as biomarkers at an individual level.

Strengths of our study are the large sample size of consecutively included patients, standardized examinations and use of a novel and validated clinimetric scale (SENS-PD). A limitation of the SENS-PD scale is that the different subdomains may reflect different systems-at-risk, indicating that similar scores between patients may reflect altogether different phenotypes, although this holds true for any multidimensional scale including the MDS-UPDRS III. From a clinical perspective, not all symptoms may be equally important, therefore the correlations with the SENS-PD scale were studied both for the composite scores and its subdomains. Our study population consisted of potential candidates for DBS. Typically, these patients had severe motor symptoms without obvious clinical signs of cognitive dysfunction or psychotic symptoms. This homogeneity constitutes both a limitation and a strength: although it is currently unknown whether our results can be generalised to all PD patients, we show that qEEG is capable of quantifying subtle differences in patients with cognitive and psychiatric symptoms that might go unnoticed in a global clinical impression. Investigations in a wider range of PD patients at different disease stages are likely to show even more pronounced correlations. Consequently, QEEG biomarkers for non-dopaminergic disease severity at different disease-stages could have clinical applicability beyond DBS screening, such as monitoring of disease progression.

The two applied methods of qEEG analysis do not show identical correlations with non-dopaminergic domains, suggesting that they address different functional aspects. The negative association of cortical connectivity in the $\alpha 2$ band with cognitive impairment but the lack of correlation with the other nonmotor domains may reflect the subcortical nature of the latter. Whereas $\alpha$-band connectivity is associated with cognition, it is not specifically limited to cognition as evidenced by a non-significant trend in the domain 'psychotic symptoms.' Although subcortical networks influence cortical processes (Boon et al. , 2017), the exact subcortical alterations are not properly visualized by EEG 
which accounts for the different results. Likewise, the lack of correlation between motor functioning and EEG slowing provides further evidence that severity of motor impairment as assessed by the MDS-UPDRS III is not reflected by cortical slowing (Babiloni et al. , 2011). Indeed, oscillatory alterations in the (subcortical) basal ganglia, which correlate with motor dysfunction (Brittain et al., 2014), cannot be captured by EEG. Magnetoencephalography (MEG) could investigate these subcortical networks (Boon et al. , 2017), however MEG is not widely available and is less applicable for routine DBS screening purposes. Previous longitudinal studies using MEG have identified slowing of oscillatory brain activities to be correlated with global disease progression in PD (Olde Dubbelink et al. , 2013).

A limitation of the applicability of EEG as a biomarker is the proportion of excluded patients due to EEG artifacts. These artifacts are partly inherent to the disease itself, such as altered muscle tone, increased ocular movements, tremor, dyskinesias, and sleepiness. As baseline characteristics, both motor and non-motor severity, did not differ between included and excluded patients, we expect that the incidence of artifacts is not associated with disease severity. This is also confirmed by the fact that in our cohort the number of recordings excluded because of artefacts was comparable in the low disease severity group and high disease severity group.

Several factors might influence EEG activity, such as aging or use of psychoactive medication. For this reason we corrected our results for both factors. Careful consideration of normal age-related alterations cannot be neglected when assessing individual EEGs. Changes of spectral- and connectivity parameters have also been found in epilepsy (Liang et al. , 2010), AD (Engels et al. , 2015), and schizophrenia (John et al. , 2009) but it remains unclear whether these disorders have a common cortical denominator or whether there are different disease-specific network alterations.

EEG is available in every hospital, readily accessible and cheaper than other functional neuroimaging measures such as MEG, fMRI, PET or SPECT (Lystad et al. , 2009). Moreover, source referencing amplified the spatial resolution, overcoming criticism of EEG's limited resolution (Burle et al., 2015). Whereas the 21-channel EEG setup could be enhanced by using a high-density setup, we demonstrated that conventional routine EEG is sufficient to provide parameters of non-dopaminergic 
disease severity. Advanced setups do not seem necessary to achieve sufficient discriminative ability on a group-level. In this study we demonstrate that disease severity is accurately reflected by both functional connectivity and simpler EEG spectral measures, which are calculated with relative ease by neurophysiologists. Future research could focus on validation in larger and more heterogeneous cohorts to investigate whether qEEG may serve as biomarkers at an individual level, and determine its accuracy in estimating disease severity. The use of EEG parameters to aid the selection process of DBS candidates should also be further investigated.

In conclusion, we have demonstrated that both EEG slowing and reduced functional connectivity in the $\alpha 2$ band are associated with increased non-dopaminergic disease severity in PD, particularly with cognitive impairment and psychotic symptoms. These EEG alterations were apparent both globally and over separate brain regions. The studied qEEG parameters may have the potential to ultimately serve as complementary biomarkers of non-dopaminergic disease severity in PD.

\section{Authors disclosures and conflicts of interest}

This work was supported by a grant from the 'Stichting ParkinsonFonds' and the 'Stichting Alkemade-Keuls'. The funding sources had no role in the study design, data collection, analysis, interpretation, writing or decision to submit the manuscript for publication. None of the authors has potential conflicts of interest that relate to this manuscript.

\section{Acknowledgements}

The authors would like to thank G.E.L. Hendriks, R.H.A.M. Reijntjes, F.I. Kerkhof and the EEG technicians of the LUMC, for their help with the data collection.

\section{References}

Ahlskog JE, Muenter MD. Frequency of levodopa-related dyskinesias and motor fluctuations as estimated from the cumulative literature. Mov Disord. 2001;16:448-58.

Babiloni C, De Pandis MF, Vecchio F, Buffo P, Sorpresi F, Frisoni GB, et al. Cortical sources of resting state electroencephalographic rhythms in Parkinson's disease related dementia and Alzheimer's disease. Clinical Neurophysiology. 2011;122:2355-64. 
Boon LI, Hillebrand A, Olde Dubbelink KTE, Stam CJ, Berendse HW. Changes in resting-state directed connectivity in cortico-subcortical networks correlate with cognitive function in Parkinson's disease. Clin Neurophysiol. 2017;128:1319-26.

Brittain JS, Brown P. Oscillations and the basal ganglia: motor control and beyond. Neuroimage. 2014;85 Pt 2:637-47.

Burle B, Spieser L, Roger C, Casini L, Hasbroucq T, Vidal F. Spatial and temporal resolutions of EEG: Is it really black and white? A scalp current density view. Int J Psychophysiol. 2015;97:210-20.

Caviness JN, Hentz JG, Belden CM, Shill HA, Driver-Dunckley ED, Sabbagh MN, et al. Longitudinal EEG changes correlate with cognitive measure deterioration in Parkinson's disease. J Parkinsons Dis. 2015;5:117-24.

Contarino MF, Daniele A, Sibilia AH, Romito LM, Bentivoglio AR, Gainotti G, et al. Cognitive outcome 5 years after bilateral chronic stimulation of subthalamic nucleus in patients with Parkinson's disease. J Neurol Neurosurg Psychiatry. 2007;78:248-52.

Cozac VV, Gschwandtner U, Hatz F, Hardmeier M, Ruegg S, Fuhr P. Quantitative EEG and Cognitive Decline in Parkinson's Disease. Parkinsons Dis. 2016;2016:9060649.

Deuschl G, Agid Y. Subthalamic neurostimulation for Parkinson's disease with early fluctuations: balancing the risks and benefits. Lancet Neurol. 2013;12:1025-34.

Duckworth AL, Yeager DS. Measurement Matters: Assessing Personal Qualities Other Than Cognitive Ability for Educational Purposes. Educ Res. 2015;44:237-51.

Duncan JS. Conventional and clinimetric approahces to individualization of antiepileptic drug therapy. In: Meinardi HC, J. A.; Baker, G. A.; da Silva A. M., editor. Quantitative assessment in epilepsy care. Porto, Portugal: Springer Science+Business Media, LLC; 1993.

Engels MM, Stam CJ, van der Flier WM, Scheltens $P$, de Waal $H$, van Straaten EC. Declining functional connectivity and changing hub locations in Alzheimer's disease: an EEG study. BMC Neurol. 2015;15:145.

George JS, Strunk J, Mak-McCully R, Houser M, Poizner H, Aron AR. Dopaminergic therapy in Parkinson's disease decreases cortical beta band coherence in the resting state and increases cortical beta band power during executive control. Neurolmage Clinical. 2013;3:261-70.

Goetz CG, Fahn S, Martinez-Martin P, Poewe W, Sampaio C, Stebbins GT, et al. Movement Disorder Society-sponsored revision of the Unified Parkinson's Disease Rating Scale (MDS-UPDRS): Process, format, and clinimetric testing plan. Mov Disord. 2007;22:41-7.

Hjorth B. Source derivation simplifies topographical EEG interpretation. American Journal of EEG Technology. 1980;20:121-32.

Jellinger KA. Neuropathology of sporadic Parkinson's disease: evaluation and changes of concepts. Mov Disord. 2012;27:8-30.

John JP, Rangaswamy M, Thennarasu K, Khanna S, Nagaraj RB, Mukundan CR, et al. EEG power spectra differentiate positive and negative subgroups in neuroleptic-naive schizophrenia patients. The Journal of neuropsychiatry and clinical neurosciences. 2009;21:160-72.

Liang SF, Wang HC, Chang WL. Combination of EEG Complexity and Spectral Analysis for Epilepsy Diagnosis and Seizure Detection. Eurasip J Adv Sig Pr. 2010. DOI 10.1155/2010/853434

Lystad RP, Pollard $\mathrm{H}$. Functional neuroimaging: a brief overview and feasibility for use in chiropractic research. The Journal of the Canadian Chiropractic Association. 2009;53:59-72.

Moldovan AS, Groiss SJ, Elben S, Sudmeyer M, Schnitzler A, Wojtecki L. The treatment of Parkinson's disease with deep brain stimulation: current issues. Neural Regen Res. 2015;10:1018-22.

Olde Dubbelink KT, Stoffers D, Deijen JB, Twisk JW, Stam CJ, Berendse HW. Cognitive decline in Parkinson's disease is associated with slowing of resting-state brain activity: a longitudinal study. Neurobiol Aging. 2013;34:408-18.

Postuma RB, Berg D, Stern M, Poewe W, Olanow CW, Oertel W, et al. MDS clinical diagnostic criteria for Parkinson's disease. Mov Disord. 2015;30:1591-601. 
Russmann H, Ghika J, Villemure JG, Robert B, Bogousslavsky J, Burkhard PR, et al. Subthalamic nucleus deep brain stimulation in Parkinson disease patients over age 70 years. Neurology. 2004;63:1952-4.

Stam CJ, Nolte G, Daffertshofer A. Phase lag index: assessment of functional connectivity from multi channel EEG and MEG with diminished bias from common sources. Hum Brain Mapp. 2007;28:117893.

Teeple RC, Caplan JP, Stern TA. Visual hallucinations: differential diagnosis and treatment. Prim Care Companion J Clin Psychiatry. 2009;11:26-32.

Tripoliti E, Zrinzo L, Martinez-Torres I, Frost E, Pinto S, Foltynie T, et al. Effects of subthalamic stimulation on speech of consecutive patients with Parkinson disease. Neurology. 2011;76:80-6. Utianski RL, Caviness JN, van Straaten ECW, Beach TG, Dugger BN, Shill HA, et al. Graph theory network function in parkinson's disease assessed with electroencephalography. Clinical Neurophysiology. 2016;127:2228-36.

van der Heeden JF, Marinus J, Martinez-Martin P, van Hilten JJ. Evaluation of severity of predominantly non-dopaminergic symptoms in Parkinson's disease: The SENS-PD scale. Parkinsonism Relat Disord. 2016;25:39-44.

Weaver FM, Follett K, Stern M, Hur K, Harris C, Marks WJ, et al. Bilateral Deep Brain Stimulation vs Best Medical Therapy for Patients With Advanced Parkinson Disease A Randomized Controlled Trial. Jama-J Am Med Assoc. 2009;301:63-73.

Zheng L, Chai H, Yu S, Xu Y, Chen W, Wang W. EEG theta power and coherence to octave illusion in first-episode paranoid schizophrenia with auditory hallucinations. Psychopathology. 2015;48:36-46.

\section{Legends}

Figure 1. Distribution of the mean spectral powers per tertile of disease severity

The high SENS-PD group demonstrated higher power in the slow EEG frequency bands (i.e. $\delta$ and $\theta$ ); the low SENS-PD group demonstrated higher power in the faster EEG frequency bands (i.e. $\alpha 1, \alpha 2$ and $\beta$ ), indicating a global slowing of EEG frequency with increasing disease severity (yellow: high power, blue: low power).

Figure 2. Correlation of slow-over-fast spectral ratio and disease severity Asterisks indicate significant correlations. A spectral ratio of slow-over-fast EEG power correlated significantly with the SENS-PD composite score (i.e. more slow EEG power correlated with increased non-dopaminergic disease severity). The subdomains psychotic symptoms and cognition correlated significantly with the spectral ratio, whilst a trend could be observed for the subdomains PIGD and depression. Error bars reflect $95 \%$ confidence intervals. 
Figure 3A. Connectivity density per tertile of non-dopaminergic disease severity (SENS-PD)

Blue: low SENS-PD group, red: intermediate SENS-PD group, green: high SENS-PD group. The high

SENS-PD group demonstrated lower $\alpha 2$ PLI density than the low SENS-PD group, indicating a at three thresholds, i.e. $P L I=0.13,0.15$ and 0.17 are shown in figure $3 B$.

Figure 3B. Average network maps of the mean PLI in the $\alpha 2$ band per tertile of disease severity

The $\alpha 2$ PLI network maps are plotted at three thresholds: $P L I=0.15,0.17$ and 0.19. A line indicates a level of functional connectivity of at least the threshold-value. With higher SENS-PD scores, the threshold for functional connectivity is lowered, indicating a greater degree of cortical

417 desynchronization with increased disease severity.

Supplementary figure 1. Correlation of global PLI values per frequency band and disease severity Error bars reflect $95 \%$ confidence intervals.

420 Supplementary figure 2. Correlation of PLI in the $\alpha 2$ band and disease severity

421 Error bars reflect 95\% confidence intervals. 
Table 1 Demographic and clinical characteristics

\begin{tabular}{|c|c|c|c|c|c|}
\hline & Overall & Low SENS-PD & Intermediate SENS-PD & High SENS-PD & $\mathrm{P}$ \\
\hline $\mathrm{N}$ & 63 & 17 & 25 & 21 & \\
\hline EEG data, seconds & 516.1 & 139.3 & 204.8 & 172.0 & \\
\hline Age, years & $62.4(7.2)$ & $61.6(6.4)$ & $62.2(7.5)$ & $63.3(7.5)$ & 0.825 \\
\hline Female sex & $20(32)$ & $6(35)$ & $8(32)$ & $6(29.0)$ & 0.906 \\
\hline Disease duration, years & $11.9(6.3)$ & $10.6(3.0)$ & $12.0(6.7)$ & $12.9(7.6)$ & 0.718 \\
\hline Use of psychoactive drugs & $15(31)$ & $3(18)$ & $5(20)$ & $7(33)$ & 0.448 \\
\hline MDS-UPDRS III total score & $24.0(10.1)$ & $20.7(9.2)$ & $24.3(11.1)$ & $26.4(9.1)$ & 0.167 \\
\hline SENS-PD total score & $13.1(4.9)$ & $7.6(1.8)$ & $12.0(0.8)$ & $18.8(2.9)$ & $<0.001^{\mathrm{a}}$ \\
\hline PIGD subscore & $1.2(1.0)$ & $0.7(0.8)$ & $1.0(1.0)$ & $1.8(0.8)$ & $0.001^{b, c}$ \\
\hline EDS subscore & $2.7(1.8)$ & $1.8(1.3)$ & $2.1(1.5)$ & $4.1(1.6)$ & $<0.001^{\text {b, }}$ c \\
\hline Depression subscore & $2.4(2.0)$ & $1.1(1.6)$ & $2.1(1.7)$ & $3.6(2.1)$ & $0.001^{b, c}$ \\
\hline Autonomic dysfunction subscore & $2.1(1.2)$ & $1.4(1.1)$ & $2.0(1.0)$ & $2.8(1.2)$ & $\mathbf{0 . 0 0 2 ^ { \mathrm { c } }}$ \\
\hline Psychotic symptoms subscore & $1.0(0.9)$ & $0.5(0.5)$ & $0.9(0.7)$ & $1.5(1.1)$ & $<0.001^{b, c}$ \\
\hline Cognition subscore & $4.0(1.6)$ & $3.0(1.6)$ & $4.0(1.2)$ & $4.8(1.6)$ & $\mathbf{0 . 0 0 2 ^ { \mathrm { c } }}$ \\
\hline
\end{tabular}

Results are expressed as mean (SD) for continuous variables, $\mathrm{n}(\%)$ for categorical variables.

${ }^{a}$ Sig. difference between all three groups. ${ }^{b}$ sig. difference between intermediate and high tertiles. ${ }^{c}$ sig. difference between low and high tertiles

PIGD: postural instability and gait difficulty; EDS: excessive daytime sleepiness 


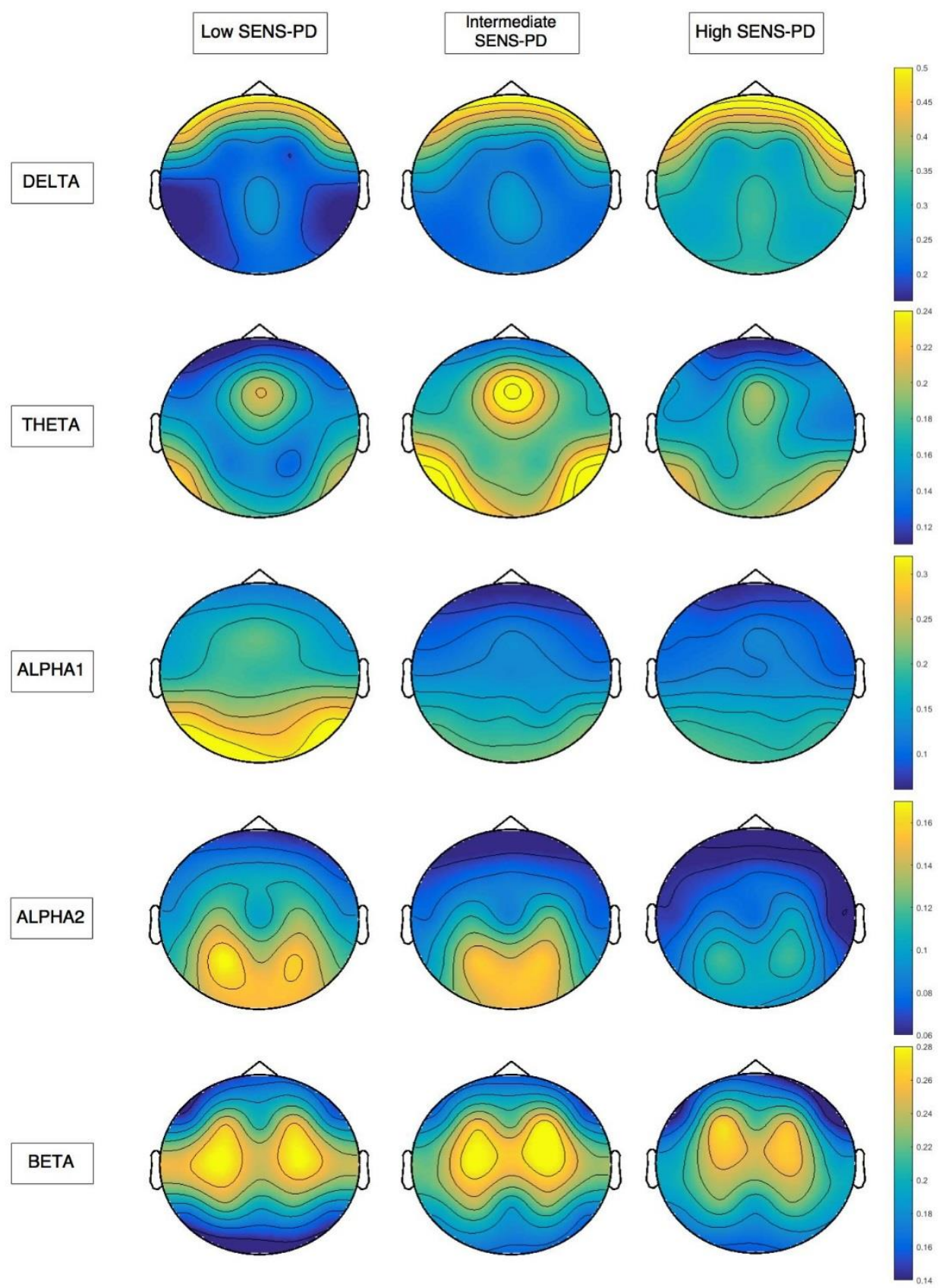

Fig 1. 




Fig 2. 


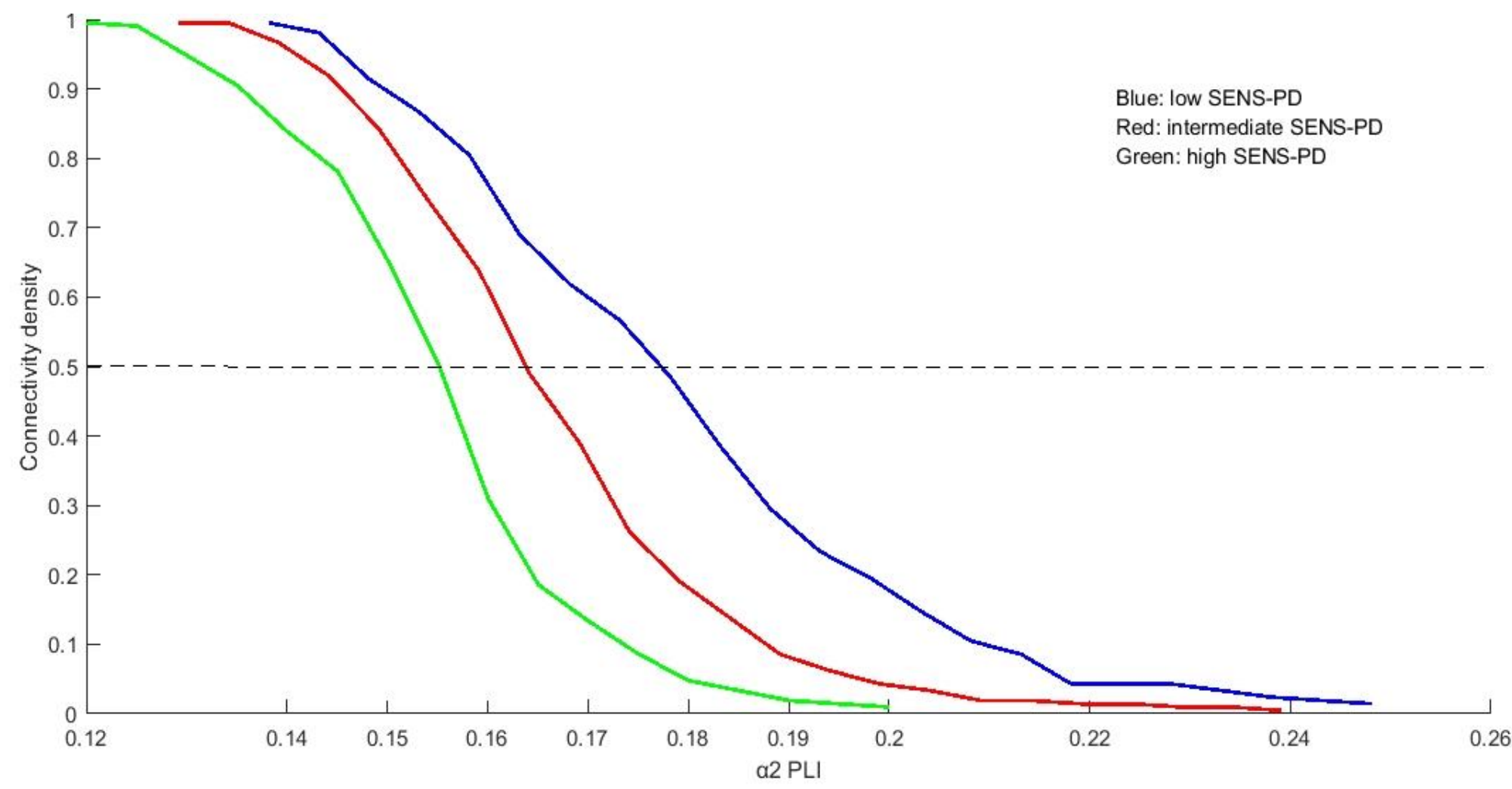



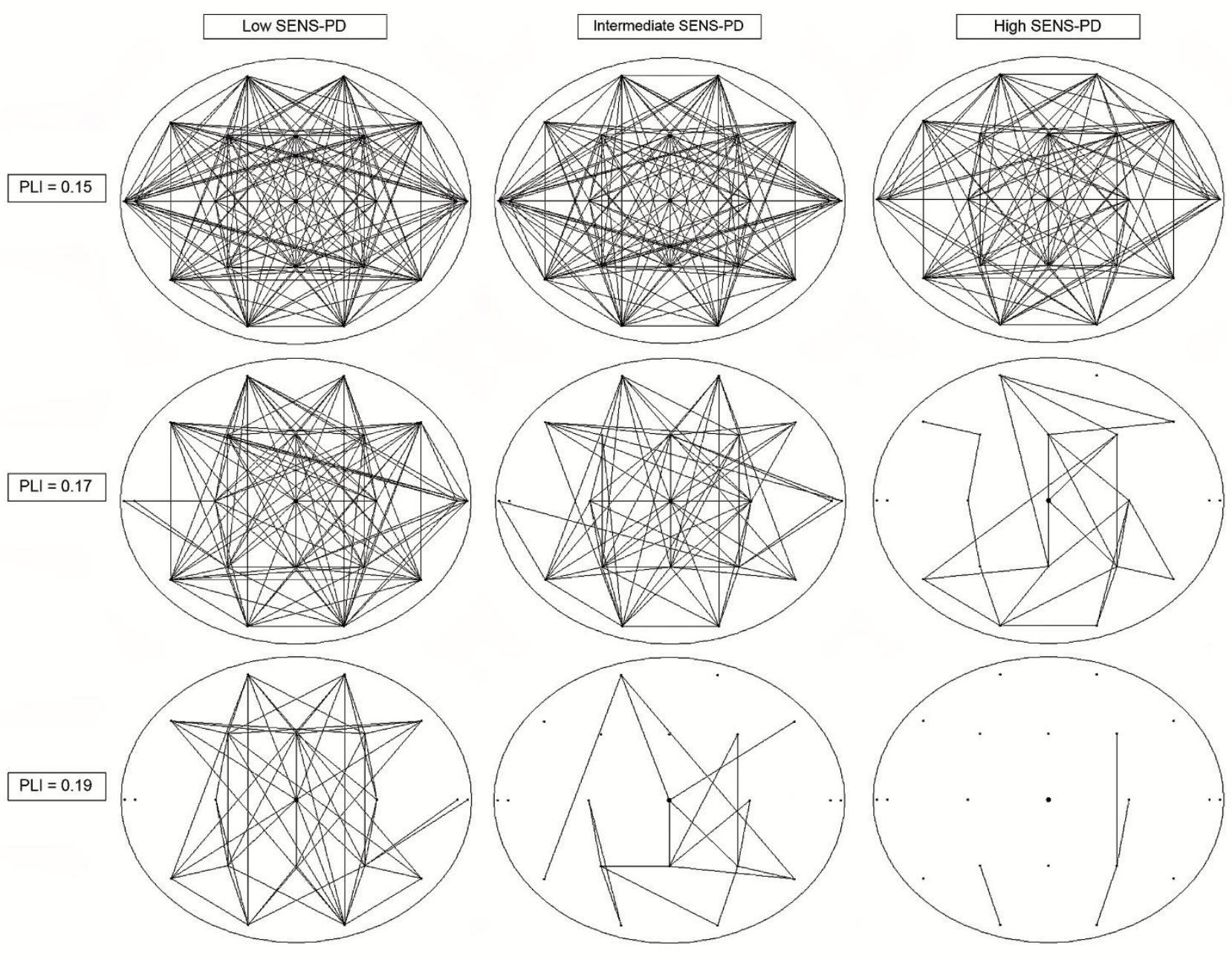

Fig 3B. 




Supp. Fig 1. 




Supp. Fig 2. 